\title{
The status of new fragment separator ACCULINNA-2 project and the first day experiments
}

\author{
S.A. Krupko ${ }^{\text {1a }}$, A.S. Fomichev ${ }^{1}$, L.V. Grigorenko ${ }^{1,4,5}$, M.S. Golovkov ${ }^{1}$, S.V. Stepantsov ${ }^{1}$, \\ G.M. Ter-Akopian ${ }^{1}$, A.A. Bezbakh ${ }^{1}$, V. Chudoba ${ }^{1,2}$, I.A. Egorova ${ }^{3}$, S.N. Ershov ${ }^{3}$, \\ A.V. Gorshkov ${ }^{1}$, V.A. Gorshkov ${ }^{1}$, G. Kaminski ${ }^{1,6}$, A.A. Korsheninnikov ${ }^{5}$, E.A. Kuzmin ${ }^{5}$, \\ E.Yu. Nikolskii ${ }^{5,7}$, Yu.Ts. Oganessian ${ }^{1}$, Yu.L. Parfenova ${ }^{1,8}$, P.G. Sharov ${ }^{1}$, S.I. Sidorchuk ${ }^{1}$, \\ R.S. Slepnev ${ }^{1}$, R. Wolski ${ }^{1,6}$, A.A. Yukhimchuk ${ }^{9}$, M.V. Zhukov ${ }^{10}$
}

${ }^{1}$ Flerov Laboratory of Nuclear reactions JINR, RU-141980 Dubna, Russia

${ }^{2}$ Institute of Physics, Silesian University in Opava, Bezručovo nám. 13, 74601 Czech Republic

${ }^{3}$ Bogolyubov Laboratory of Theoretical Physics, JINR, Dubna, RU-141980 Russia

${ }^{4}$ GSI Helmholtzzentrum für Schwerionenforschung, planckstraße 1, D-64291 Darmstadt, Germany

${ }^{5}$ Russian Research Center "The Kurchatov Institute", Kurchatov sq. 1, RU-123182 Moscow, Russia

${ }^{6}$ Institute of Nuclear Physics PAN, Radzikowskiego 152, PL-31342 Kraków, Poland

${ }^{7}$ RIKEN, Hirosawa 2-1, Wako, Saitama 351-0198, Japan

${ }^{8}$ Skobeltsyn Institute of Nuclear Physics, Moscow State University, 119991 Moscow, Russia

${ }^{9}$ RNFC - All-Russian Research Institute of Experimental Physics, RU-607190, Sarov, Nizhni

Novgorod Region, Russia

${ }^{10}$ Fundamental Physics, Chalmers University of Technology, S-41296 Göteborg, Sweden

\begin{abstract}
The new facility fragment separator ACCULINNA-2 will be put into operation at the beginning of 2015 in FLNR JINR. The new separator is destined to add considerably to the studies of drip-line nuclei performed with the use of variety of direct reactions known to be distinctive to the $15-50 \mathrm{MeV} / \mathrm{amu}$ exotic secondary RIBs. Intense beams provided by the U-400M cyclotron will ensure the achievement of this objective. In addition to the RIB separation accomplished by means of the dipole-wedge-dipole selection, the addition of a zero-degree dipole magnet setup is foreseen. A long (13 $\mathrm{m})$ straight section will provide precise time-of-flight measurements.
\end{abstract}

\section{Fragment separator ACCULINNA-2}

FLNR JINR project ACCULINNA-2 [1] is a new facility - fragment separator, producing secondary RIBs for nuclear physics experiments. High intensity, DC mode primary beam of U-400M cyclotron hits the solid beryllium, rotated liquid-cooled production target. Warm magnets including sextupole and octupole elements are used to form the ion-optical separator line. RIB separation is accomplished by means of dipole-wedge-dipole selection. Low intensity secondary part of separator is placed outside the accelerator closed area providing good background conditions in the experimental area.

${ }^{\mathrm{a} C}$ Corresponding author: krupko@jinr.ru

This is an Open Access article distributed under the terms of the Creative Commons Attribution License 2.0, which permits unrestricted use, distribution, and reproduction in any medium, provided the original work is properly cited. 
ACCULINNA-2 will upgrade the system of ACCULINNA to a new quality of secondary beams: rising of the RIB intensities is 10 times and more; beam purity will be higher, especially for protonrich RIBs; quite precise ToF measurement will be provided by a $13-\mathrm{m}$ flight base; zero-degree spectrometer for registration of beam-like reaction products will be added soon after the startup of the main separator. Primary beams provided by the U-400M cyclotron could produce the following RIBs, as shown in Table 1.

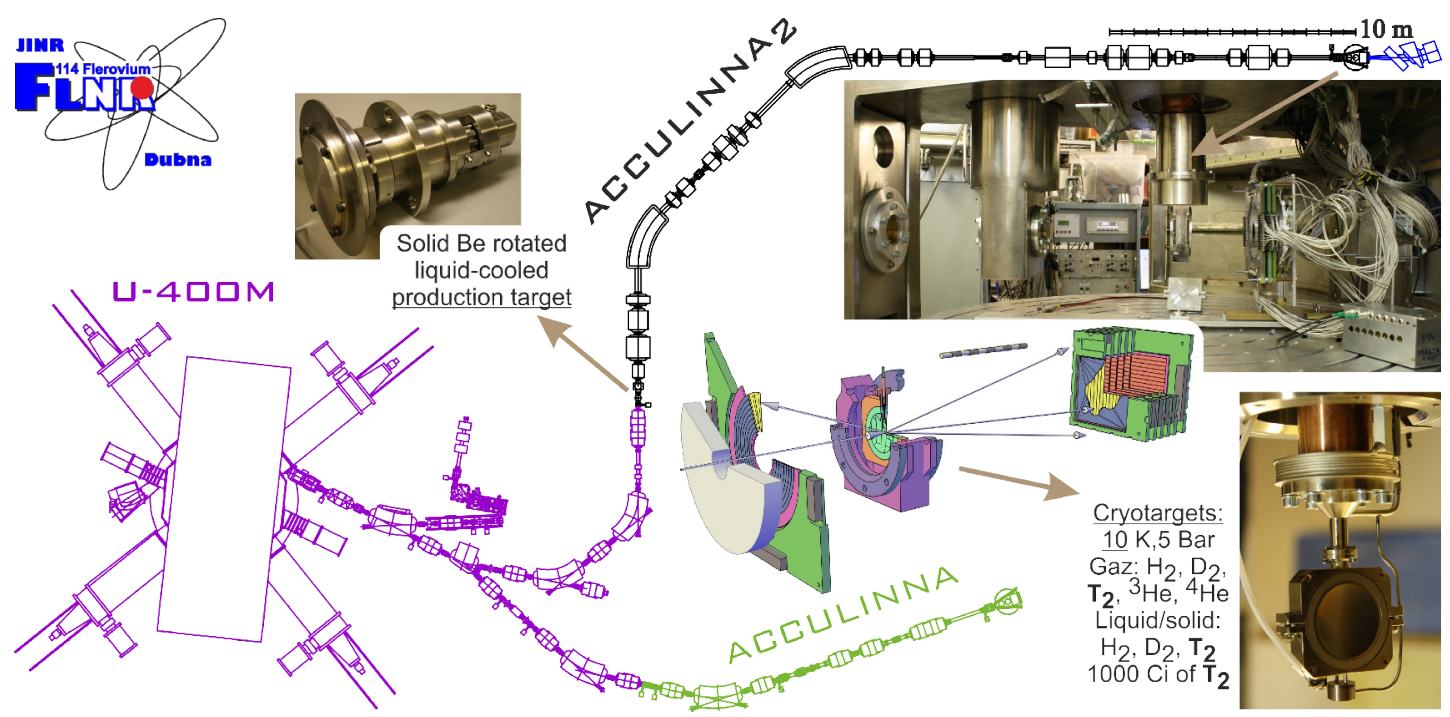

Figure 1. ACCULINNA, ACCULINNA-2 layout and experimental setup

Zero-degree spectrometer should be included into start version. The developed spectrometer will cover $\pm 2.8^{\circ}$ acceptance for the beam-like reaction products with a $1 \mathrm{~mm}$ in/out position accuracy and with ToF identification. The commissioning of the separator is planned in 2014, the first experiments are to be carried out in 2015 .

Table 1. Expected secondary beams of ACCULINNA- 2 without RF-kicker. Acceptance: $\Delta B \rho / B \rho= \pm 2 \%, \Delta \theta x=$ $\Delta \theta y= \pm 30 \mathrm{mrad}, \mathrm{F} 3-\mathrm{Slit}=10 \mathrm{~mm}$

\begin{tabular}{|c|c|c|c|c|c|c|}
\hline Primary beam & $\begin{array}{c}\mathbf{E}_{\text {beam, }} \\
\mathbf{A M e V}\end{array}$ & $\begin{array}{c}\mathbf{I}_{\text {beam, }} \\
\mathbf{p} \boldsymbol{\mu} \mathbf{A}\end{array}$ & $\mathbf{R I B}$ & $\begin{array}{c}\mathbf{E} \mathbf{W}_{\mathbf{R I B}}, \\
\mathbf{A M e V}\end{array}$ & $\begin{array}{c}\mathbf{I}_{\text {RIB }}, \\
\mathbf{p p s}\end{array}$ & $\begin{array}{c}\text { Purity } \\
\text { \% }\end{array}$ \\
\hline${ }^{7} \mathrm{Li}^{2+}$ & 34.0 & 4 & ${ }^{6} \mathrm{He}$ & 28 & $\mathbf{3 . 5 \times 1 0 ^ { 7 }}$ & 99 \\
\hline${ }^{11} \mathrm{~B}^{3+}$ & 33.0 & 10 & ${ }^{8} \mathrm{He}$ & 24.5 & $\mathbf{2 . 8 \times 1 0 ^ { 5 }}$ & 99 \\
\hline- & - & - & ${ }^{9} \mathrm{Li}$ & 23.5 & $\mathbf{1 . 5 \times 1 0 ^ { 7 }}$ & 99 \\
\hline${ }^{15} \mathrm{~N}^{5+}$ & 47.0 & 10 & ${ }^{8} \mathrm{He}$ & 35 & $\mathbf{1 . 4 \times 1 0 ^ { 5 }}$ & 99 \\
\hline- & - & - & ${ }^{11} \mathrm{Li}$ & 38 & $\mathbf{2 . 0 \times 1 0 ^ { 4 }}$ & 93 \\
\hline${ }^{18} \mathrm{O}^{6+}$ & 51.0 & 10 & ${ }^{11} \mathrm{Li}$ & 40 & $\mathbf{2 . 5 \times 1 0 ^ { 4 }}$ & 90 \\
\hline- & - & - & ${ }^{14} \mathrm{Be}$ & 42 & $\mathbf{5 . 8 \times 1 0 ^ { 3 }}$ & 98 \\
\hline- & - & - & ${ }^{15} \mathrm{~B}$ & 41 & $\mathbf{1 . 6 \times 1 0 ^ { 6 }}$ & 97 \\
\hline${ }^{36} \mathrm{~S}^{11+}$ & 40.6 & 10 & ${ }^{24} \mathrm{O}$ & 23 & $\mathbf{7 . 7 \times 1 0 ^ { 3 }}$ & 9 \\
\hline- & - & - & ${ }^{17} \mathrm{~B}$ & 21.5 & $\mathbf{2 . 2 \times 1 0 ^ { 3 }}$ & 10 \\
\hline${ }^{10} \mathrm{~B}^{3+}$ & 40.0 & 10 & ${ }^{8} \mathrm{~B}$ & 26.5 & $\mathbf{3 . 6 \times 1 0 ^ { 7 }}$ & 45 \\
\hline${ }^{20} \mathrm{Ne}^{7+}$ & 54.5 & 10 & ${ }^{13} \mathrm{O}$ & 31.5 & $\mathbf{3 . 0 \times 1 0 ^ { 6 }}$ & 8 \\
\hline
\end{tabular}




\begin{tabular}{|c|c|c|c|c|c|c|}
\hline Primary beam & $\begin{array}{c}\mathbf{E}_{\text {beam, }} \\
\mathbf{A M e V}\end{array}$ & $\begin{array}{c}\mathbf{I}_{\text {beam, }} \\
\mathbf{p} \boldsymbol{\mu} \mathbf{A}\end{array}$ & $\mathbf{R I B}$ & $\begin{array}{c}\mathbf{E w}_{\text {RIB }}, \\
\mathbf{A M e V}\end{array}$ & $\begin{array}{c}\mathbf{I}_{\text {RIB }}, \\
\mathbf{p p s}\end{array}$ & $\begin{array}{c}\text { Purity } \\
\mathbf{\%}\end{array}$ \\
\hline- & - & - & ${ }^{14} \mathrm{O}$ & 34.0 & $\mathbf{7 . 1 \times 1 0 ^ { 7 }}$ & 45 \\
\hline- & - & - & ${ }^{17} \mathrm{Ne}$ & 32.5 & $\mathbf{1 . 2 \times \mathbf { 1 0 } ^ { 7 }}$ & 75 \\
\hline${ }^{32} \mathrm{~S}^{11+}$ & 51.4 & 10 & ${ }^{24} \mathrm{Si}$ & 25.0 & $\mathbf{5 . 9 \times 1 0 ^ { 4 }}$ & 1.2 \\
\hline- & - & - & ${ }^{27} \mathrm{~S}$ & 23 & $\mathbf{1 . 3 \times \mathbf { 1 0 } ^ { 3 }}$ & 0.5 \\
\hline
\end{tabular}

With its quite a short ion-optical line ACCULINNA-2 will allow to detect short-lived (down to $150 \mathrm{~ns}$ ) exotic nuclei emerging straight from the production target. The RIB energy range $15-50 \mathrm{MeV} / \mathrm{amu}$ is not wide spread, but well suited for the production and study of drip-line nuclei obtained in charge-exchange and transfer reactions taking place in bombardments of light targets (e.g. hydrogen, deuterium, tritium, helium-3 etc.) with the ACCULINNA-2 secondary beams.

\section{Instrumentation}

Various kinds of instruments are being developed and/or are already applied in the current experiments performed at the RIBs of the working ACCULINNA separator. A typical setup consists of the RIB diagnostic ToF and Multi Wire Proportional Chambers (MWPC), cryogenic gas/liquid target, charged particle telescopes, and, optionally, neutron detectors wall, $\gamma$-ray detectors around the target.

Two identical ToF detectors, placed on both ends of the straight beam line (ACCULINNA: $8 \mathrm{~m} /$ ACCULINNA-2: $13 \mathrm{~m}$ ), provide for the RIBs the energy-loss and time-of-flight measurements, eventby-event. The dE-ToF method and time measurements with amplitude correction and absolute calibration provide energy resolution not worse than $0,5 \%$ (FWHM) and robust ion identification at count rate up-to $5 \mathrm{MHz}$.

Each detector consists of thin organic scintillator, typically $100-250 \mu \mathrm{m}$ thick and 2 or 4 PMTs, placed perpendicular to the beam and catching the light coming out from the scintillator. The signals are split to leading edge discriminator and QDC with fast amplification if needed.

Beam tracking done by two MWPCs grants the determination of the hit position of individual RIBs on the physics target with a 2-mm accuracy and limited count rate of $1 \mathrm{MHz}$.

Cryogenic physics targets have been successfully used in all key experiments at ACCULINNA, for example, a unique tritium target device is described in [2]. Target cells with thin stainless steel foil windows $\varnothing 20 \mathrm{~mm}$ have good ratio of active/dead material and in/out aperture not less $\pm 45^{\circ}$, working temperature down to $10 \mathrm{~K}$ for rising density and changing state of matter.

Table 2. Available physical targets for ACCULINNA/ACCULINNA-2

\begin{tabular}{|c|c|c|c|c|c|}
\hline Working gas & Phase & $\begin{array}{c}\text { Cell thickness, } \\
\text { mm }\end{array}$ & $\begin{array}{l}\text { Windows, SS } \\
\text { foils }\end{array}$ & Pressure, bar & $\begin{array}{c}\text { Max thickness, } \\
\text { atoms } / \mathbf{c m}^{2}\end{array}$ \\
\hline $\mathrm{H}_{2}, \mathrm{D}_{2},{ }^{3} \mathrm{He},{ }^{4} \mathrm{He}$ & Gas & $4-6$ & $2 \mathrm{x}(6-13) \mu \mathrm{m}$ & 5 & $2 \times 10^{21}$ \\
\hline $\mathrm{H}_{2}, \mathrm{D}_{2}$ & Liquid / solid & $0.4-0.6$ & $2 \times 6 \mu \mathrm{m}$ & $0.5-1.5 / 0.2$ & $2.5 \times 10^{21}$ \\
\hline $\mathrm{T}_{2}$ & Gas / Liquid & $4-6 / 0.4-0.6$ & $4 \mathrm{x} 9 \mu \mathrm{m}$ & $0.9 / 0.5-0.9$ & $1 \times 10^{21}$ \\
\hline
\end{tabular}

Two geometries for charged particle detector telescopes are being used: coaxial with hole in center and square shaped. For both geometries a wide range of detectors is available for covering full path of lightest particles with the accessible energy, see Table 3. A few telescopes can be combined on the axis and/or in plane to accept the necessary angles.

The study of neutron-rich nuclei can be completed with neutron detection provided by an array of 32 monocrystal scintillator stilbene detectors. Crystals $\varnothing 80 \times 50 \mathrm{~mm}^{3}$ with PMT and related electronics are packed in compact, $\varnothing 107 \mathrm{~mm}$, steel shield. The threshold for $\mathrm{n}-\gamma$ discrimination $400 \mathrm{keV}$ in 
neutron scale (55 keV for electrons) is obtained in total vs. slow QDC integrals comparison. Time resolution better $1 \mathrm{~ns}$ allows to reduce ToF base for neutrons to $1.5-2 \mathrm{~m}$.

Data acquisition system combines typically 200 parameters of ToF, MWPC, Si and CsI(Tl) for charged particles, neutron and $\gamma$-ray detectors in VME standard with $3 \mathrm{kHz}$ accepted triggers. This solution is described in [3].

Table 3. Available detectors for charge particles

\begin{tabular}{|c|c|c|c|c|}
\hline Geometry & $\begin{array}{c}\text { Active size, } \\
\text { mm }\end{array}$ & $\begin{array}{l}\text { Strip structure, } \\
\text { Front/Back }\end{array}$ & $\begin{array}{c}\text { Thickness, } \\
\mu \mathrm{m}\end{array}$ & Array CsI(Tl) \\
\hline \multirow{3}{*}{ coaxial } & \multirow{2}{*}{$\varnothing 84$ / Ø33 hole } & 32 sectors / 1 & 1000 & \multirow{3}{*}{$\begin{array}{c}\varnothing 86 \text { / Ø31 hole } \\
16 \text { sectors } \\
\text { Thickness: } \\
18 \text { or } 25 \mathrm{~mm}\end{array}$} \\
\hline & & 32 sectors / 32 rings & 300,1000 & \\
\hline & $1 / 8\left(45^{\circ}\right)$ of $\varnothing 70$ / Ø26 hole & $1 / 1$ & $40-45$ & \\
\hline \multirow{4}{*}{ square } & \multirow{2}{*}{$62 \times 62$} & $32 \mathrm{X} / 1$ & 1000 & \multirow{4}{*}{$\begin{array}{c}65 \times 65 \mathrm{~mm}^{2} \\
4 \times 4 \text { square } \\
\text { Thickness: } \\
30 \mathrm{~mm}\end{array}$} \\
\hline & & $32 \mathrm{X} / 32 \mathrm{Y}$ & 300,1000 & \\
\hline & $58 \times 58$ & $16 \mathrm{X} / 1$ & 22,65 & \\
\hline & $40 \times 40$ & $40 \mathrm{X} / 40 \mathrm{Y}$ & 65 & \\
\hline
\end{tabular}

\section{Experimental program}

New results obtained in continuation of a recent ${ }^{10} \mathrm{He}$ study [4] show the potential of the (t,p) and (d,p) type reactions for precise study of heavier nuclei nearby the neutron drip line. The excitation spectra of ${ }^{11-13} \mathrm{Li},{ }^{13-16} \mathrm{Be},{ }^{16-19} \mathrm{~B}$, and ${ }^{19-22} \mathrm{C}$ will be a first-priority task for ACCULINNA-2. The search for the few-neutron radioactive decays is a challenging task requiring also application of novel experimental approaches. Quite sufficient intensities of the ${ }^{24} \mathrm{O},{ }^{26,27} \mathrm{~F},{ }^{28-30} \mathrm{Ne}$ RIBs offered by ACCULINNA-2 will be admissible for the study of resonant states of respective nuclei (e.g. $\left.{ }^{24-26} \mathrm{O}\right)$ lying near and beyond the drip line.

The choice of ACCULINNA-2 RIBs will offer especially favorable conditions for the study of nuclei beyond the proton drip line carried out with the use of the $(p, d),(p, t)$ and $\left({ }^{3} \mathrm{He}, n\right)$ reactions. Recently, the ACCULINNA group performed a dedicated search for the drip line nucleus ${ }^{26} \mathrm{~S}$ produced in fragmentation of a $50.3 \mathrm{MeV} / \mathrm{amu}{ }^{32} \mathrm{~S}$ beam [5]. An upper half-life limit of $T_{1 / 2}<79 \mathrm{~ns}$ was set for ${ }^{26} \mathrm{~S}$ in this study. Any dedicated search has not been performed yet for the neighbor nuclei ${ }^{21} \mathrm{Mg},{ }^{30} \mathrm{Ar}$, and ${ }^{34} \mathrm{Ca}$, which could exist with half-lives shorter than $100 \mathrm{ps}$. The properties of these nuclei (including also ${ }^{26} \mathrm{~S}$ ) could be ascertained well with the ACCULINNA-2 RIBs.

\section{Acknowledgments}

The authors are grateful to the project coworkers I. Mukha, M. Pfutzner, O. Tarasov and H. Simon. The work was supported by the Russian Foundation for Basic Research, project no. 11-02-00657-a. S.A.K., L.V.G., I.A.E. and A.V.G. are supported by FAIR-Russia Research Center grant.

\section{References}

1. A.S. Fomichev at al., JINR Communication E13-2008-168 (2008)

2. A.A. Yukhimchuk at al., Nucl. Instr. and Meth. in Phys. Res. A 513 (2003) 439-447

3. R.S. Slepnev at al., Instr. and Exper. Techn. MAIK Nauka/Interpeiodica 556 (2012) 645-650

4. S.I. Sidorchuk at al., Physical Review Letters 108, 202502 (2012)

5. A.S. Fomichev at al., Int. Jounal of Modern Physics E Vol. 20, No. 6 (2011) 1491-1508 